\title{
Sala de Recreación para Adolescentes Internados en un hospital de alta complejidad. Programa para la Atención Integral de Adolescentes Internados
}

\author{
A Recreation Room for adolescents who are hospitalized at a \\ tertiary-care Center. Care Program for Hospitalized Adolescents
}

\author{
Dr. Roberto Mato y Dra. M. Susana Rodríguez
}

\begin{abstract}
RESUMEN
La adolescencia es un período trascendente en el desarrollo del ser humano. Una hospitalización es un acontecimiento de alto impacto, que puede generar ansiedad, depresión y angustia, y afectar ese desarrollo en forma adversa.

En el Hospital Garrahan, donde los adolescentes constituyen más del 30\% de los pacientes, se desarrolla el Programa para la Atención Integral de los Adolescentes Internados, que, a través de diferentes intervenciones, intenta dar cabida a las necesidades de esa población. Formando parte de ese programa, la Sala de Recreación para Adolescentes Internados busca crear un entorno adecuado para atenuar el impacto de la internación y favorecer la transmisión de conocimientos, normas y pautas de comportamiento en un ámbito en el que continúan los cuidados médicos y de enfermería que el paciente necesita.

Las intervenciones que combinan aspectos médicos, recreacionales, educativos y de contención emocional son efectivas para reducir el impacto negativo de la hospitalización y para prevenir conductas de riesgo.

Elobjetivo es comunicar nuestra experiencia en la Sala de Recreación para Adolescentes Internados. Palabras clave: servicios de salud, adolescente, recreación, prevención, conducta de reducción del riesgo.
\end{abstract}

\section{ABSTRACT}

Hospital admission is a high-impact event in children. Adolescence is a critical and complex period of human development that may be adversely affected by hospitalization.

Pediatría "Prof. Dr.

J. P. Garrahan",

CABA, Argentina.

Correspondencia: Dr. Roberto Mato: robertomato@hotmail. com

\section{Financiamiento:}

Ninguno.

\section{Conflicto de intereses:}

Ninguno que declarar.

Recibido: 22-8-2014

Aceptado: 8-1-2015
At the Garrahan Hospital, where adolescents account for more than $30 \%$ of inpatients, a program for comprehensive care of adolescents was set up in 2008 with a special focus on their specific needs.

As a part of this program, the aim of the Recreation Room for Hospitalized Adolescents is to provide a friendly environment to reduce stress and anxiety and to facilitate the learning of healthy behaviors, under the permanent care of nurses and medical doctors.

Interventions in health, leisure time, education, and emotional care are effective in diminishing the negative impact of hospitalization and prevent risk behaviors.
Our objective was to report our experience in the Recreation Room for Hospitalized Adolescents. Key words: health services, adolescent, recreation, prevention, risk reduction behavior.

http:/ /dx.doi.org/10.5546/aap.2015.254

\section{INTRODUCCIÓN}

En los hospitales pediátricos de alta complejidad, la atención y el seguimiento de pacientes con patologías crónicas y complejas son responsables del aumento en la edad promedio de su población. En el Hospital de Pediatría "Prof. Dr. J. P. Garrahan", un hospital de tercer nivel de la Ciudad de Buenos Aires, el 33\% de las consultas ambulatorias y de las internaciones anuales (90 000 consultas, 7722 egresos en el año 2013) correspondieron a mayores de 10 años.

En esta etapa, caracterizada por impactantes cambios en lo físico, emocional, intelectual y social, la búsqueda de una identidad y la experimentación en distintos escenarios, es cuando irrumpen la enfermedad y la internación. Se entiende, entonces, el porqué de la alta vulnerabilidad y lo complejo de asimilar situaciones como las planteadas, en nuestro caso, en un hospital pediátrico.

La población adolescente presenta características y necesidades que no han sido contempladas en los hospitales pediátricos o generales donde se atienden. Las enfermedades crónicas impactan en el desarrollo normal de los adolescentes, prolongando su dependencia, afectando su imagen corporal y sus 
relaciones sociales, retrasando la escolaridad. El cuidado de su salud requiere también el conocimiento de aspectos éticos y legales. ${ }^{1-3}$

Cuando permanecen internados, especialmente durante períodos prolongados, manifiestan necesidades de autonomía, recreación, socialización, comunicación, que no pueden satisfacerse sin un ámbito y un equipamiento adecuados. ${ }^{4-6}$ Refieren que no se respeta su privacidad, que deben compartir la habitación con niños pequeños que los molestan, que los horarios de alimentación y sueño son muy rígidos. En lo concerniente a aspectos de prevención, solo el $1 \%$ de un grupo de adolescentes encuestados refirió haber recibido en el hospital información sobre salud sexual y reproductiva o prevención de adicciones. ${ }^{7}$

Los hospitales pediátricos, generalmente, disponen de espacios de juego apropiados para los más pequeños, que no resultan atractivos para los adolescentes. La posibilidad de salir de las salas de internación, jugar, comer en compañía, adquirir diferentes habilidades, intercambiar vivencias con sus pares y comunicarse con amigos y familiares que no están en el hospital representa un aporte importante para mejorar la calidad de su atención y ayuda a reducir el estrés generado por la internación..$^{8-10}$ No tenemos conocimiento ni hemos encontrado en la bibliografía la existencia de espacios recreativos preparados para la permanencia prolongada de niños o adolescentes con tratamientos en curso fuera de las salas de internación.

El Hospital Garrahan es un hospital de 520 camas, 308 de las cuales (en el área denominada Cuidados Intermedios y Moderados) están asignadas a la internación de pacientes con grados variables de complejidad y estabilidad, que sigue la modalidad de cuidados progresivos. Están distribuidas en 9 unidades de internación policlínicas, con cierta orientación funcional, pero sin discriminación por edad o especialidad.

A partir de 2008, comenzó a desarrollarse, en el Hospital "Prof. Dr. J. P. Garrahan”, el Programa para la Atención Integral de los Adolescentes Internados. Sus objetivos son los siguientes:

- Incorporar al equipo de atención de los pacientes internados médicos especialistas en la atención de adolescentes. Su participación se realiza en forma interdisciplinaria, ante la solicitud del médico clínico o especialista que atiende al paciente, cuando considera que se requiere una intervención especial.

- Brindar educación continua al personal asistencial y en formación sobre las características particulares de ese grupo etario en los aspectos físicos, psicológicos, patológicos y legales.

- Aprovechar la internación para brindar a los pacientes información sobre temas de educación para la salud, prevención de adicciones, prevención de embarazo adolescente y enfermedades de transmisión sexual. ${ }^{11}$ Esta información se brindará en forma de talleres, lo que buscará la participación activa de los adolescentes en un ambiente atractivo, al que concurran en forma espontánea o por invitación personalizada.

- Extender la consejería a las madres y padres adolescentes de los niños internados (aproximadamente, 1100 madres y/o padres por año).

- Crear un espacio de recreación atractivo (Sala de Recreación para Adolescentes Internados), donde puedan permanecer por períodos prolongados sin discontinuar sus tratamientos.

- Articular la transferencia para la atención en hospitales para adultos. Esta deberá realizarse en forma personalizada, anticipada, participativa, seleccionando las instituciones más adecuadas según la patología de base, accesibilidad e interés por recibir a estos pacientes.

\section{LA SALA DE RECREACIÓN PARA ADOLESCENTES INTERNADOS \\ Objetivos}

- Brindar actividades de recreación apropiadas para la edad adolescente.

- Estimular la comunicación entre pares. Favorecer la comunicación con "el afuera" a través de internet y redes sociales.

- Incluir al mayor número posible de pacientes internados.

- Incluir al mayor número posible de madres adolescentes de pacientes internados que pueden concurrir a la Sala con sus hijos enfermos.

- Brindar atención médica y de enfermería coordinada con la de las unidades de internación para permitir estancias prolongadas en la Sala de Recreación.

- Generar un ámbito propicio para transmitir contenidos de educación para la salud en general y educación sexual y reproductiva en particular.

- Proveer los insumos del Programa de Salud Sexual y Procreación Responsable del 
Ministerio de Salud de Nación y de la Ciudad Autónoma de Buenos Aires (preservativos, anticonceptivos).

\section{Población}

- Adolescentes internados de 10 o más años de edad.

- Madres y/o padres adolescentes menores de 20 años que acompañan en el Hospital a sus hijos internados.

\section{Criterios de admisión de la Sala de Recreación}

- Estabilidad hemodinámica y respiratoria.

- No estar cursando una enfermedad transmisible.

- No presentar compromiso inmune grave.

- Estar autorizado por su médico tratante.

- Concurrir preferentemente sin los padres. Las madres adolescentes pueden concurrir con sus hijos si estos cumplen los criterios médicos de admisión.

\section{Funcionamiento}

La Sala permanece operativa de 8 a18 h y no hay límite para el tiempo de permanencia.

Se estimula la concurrencia a través de los médicos tratantes y mediante una búsqueda personalizada por parte de las enfermeras de la Sala, que disponen diariamente del censo de adolescentes internados y de madres adolescentes de niños internados en el Hospital.

Los pacientes que pueden trasladarse (por sus medios o asistidos) concurren a la Sala, donde continúan recibiendo el tratamiento indicado (infusiones, oxigenoterapia, nutrición parenteral, etc.). Si el tratamiento excede las capacidades de la Sala, vuelven a su lugar de internación en el horario correspondiente. Al contar la Sala con personal de enfermería entrenado e infraestructura adecuada (pie de sueros, oxígeno, oximetría de pulso, etc.), puede continuarse con la mayoría de los tratamientos. Los pacientes que no pueden ser trasladados (por ejemplo, por razones de aislamiento) reciben, en su lugar de internación, a los integrantes del Programa de Atención de Adolescentes, quienes les proveen elementos de recreación (notebook, material gráfico, material para actividades plásticas, etc.).

\section{Ámbito físico}

La Sala está ubicada en el mismo piso que las unidades de internación.

En una superficie de $85 \mathrm{~m}^{2}$ con acceso a un balcón terraza, se dispuso un consultorio, un área médica con equipamiento para emergencias, baño, pileta, depósito y un espacio amplio multifuncional.

Posee, además, una puerta de acceso amplia para permitir el ingreso de sillas de ruedas, camillas y camas, ductos para oxígeno, múltiples tomas de corriente eléctrica (para bombas de infusión, oxímetros, equipos de recreación), interno telefónico, conexión wi-fi.

\section{Capacidad}

Es de 15 pacientes sentados que realizan diferentes actividades. En los talleres, han asistido hasta 25 personas, que, sumadas al personal del sector, lleva a 30 personas el nivel máximo de ocupación.

\section{Higiene}

Se realiza con los mismos criterios que en las unidades de internación, con especial atención a evitar la transmisión a través del contacto de las manos del personal y de fomites.

Los teclados no lavables se cubren con papel film, que se cambia entre paciente y paciente.

\section{Equipamiento}

- Computadoras con acceso a internet y redes sociales.

- Reproductor de DVD.

- Equipo de audio.

- Cañón para proyecciones.

- Mesas y sillas.

- Materiales para actividades artísticas y manuales (instrumentos musicales, pinturas, pinceles, fibras, lápices, mandalas para pintar, elementos de jardinería, etc.).

- Juegos de salón y de ingenio, libros, revistas, videos, Playstation.

\section{Personal}

El sector fue concebido como una prolongación de la internación, donde el paciente pudiera continuar sus tratamientos, mantener la oxigenoterapia, recibir infusiones, alimentaciones enterales, tracciones, etc., durante períodos prolongados.

Para cumplir este objetivo, fue imprescindible que el personal del área estuviera integrado por enfermeras capacitadas en las patologías que se atienden en el Hospital y sus terapéuticas, y un referente médico especialista en adolescencia para resolver y coordinar la atención de eventos no previstos. 


\section{Talleres}

Se ha demostrado la eficacia de utilizar las oportunidades de interacción de los adolescentes con el ámbito de la salud para realizar la prevención. ${ }^{4,11}$ Por ello, se organizó, en forma de talleres, el tratamiento de diferentes tópicos, como educación para la salud, prevención de accidentes y conductas de riesgo, adicciones, salud sexual y reproductiva, planificación familiar, proyecto de vida, la escuela, orientación vocacional, orientación laboral. Estos talleres son coordinados tanto por el personal médico como el de enfermería del sector.

\section{RESULTADOS}

En el año 2013, se produjeron en el Hospital 23463 egresos, de los cuales 7722 (el 33\%) correspondieron a pacientes de 10 años o más. El $21 \%$ de los egresos incluyó al grupo etario de 10 a 14 años; el 11\%, a jóvenes de entre 15 y 19 años; y un $1 \%$ (150 pacientes), a mayores de 19 años.

El 24\% de las madres de los niños internados en Neonatología era adolescente. En las Unidades de Cuidados Intensivos, fue el 7\%. El número total, en el año, fue de 1100 madres y/o padres adolescentes. Acorde a encuestas realizadas en diferentes momentos del desarrollo del Programa, en la planilla de registro que se confecciona previamente a la distribución de insumos y las entrevistas personales, se detecta que estas madres carecen, en su mayoría, de nociones básicas de cuidados para la salud y aspectos concernientes a salud sexual y reproductiva.

\section{Actividades realizadas}

En los primeros dos años de funcionamiento de la Sala, la concurrencia fue numerosa y entusiasta (Tabla 1), con un promedio de concurrencia de 11 pacientes por día hábil. El número de talleres se incrementó en el segundo año, con un aumento en el número global de participantes.

La experiencia con las madres adolescentes fue movilizadora. Este grupo, que muchas veces se traslada al hospital junto con su hijo en el posparto inmediato, mostró una gran necesidad de controles médicos y contención, y avidez por la adquisición de conocimientos (planificación familiar, prevención de embarazos no buscados, prevención de enfermedades de transmisión sexual).

\section{COMENTARIO}

Los objetivos principales de la Sala son mejorar la calidad de la atención brindando un espacio amigable con actividades de recreación y comunicación apreciadas por los adolescentes y trabajar en prevención.

Capitalizar los tiempos ociosos de las internaciones prolongadas y llenarlos con contenidos temáticos propios de la edad, suma y potencia los logros obtenidos en la internación. Un aspecto muy valorado por los pacientes es disponer de un acceso fluido para comunicarse y mantener vínculos con sus amigos, familia y entorno a través de internet y las redes sociales independientemente de las distancias que los separan.

La característica distintiva de la Sala es el equipamiento y la presencia de personal médico y de enfermería entrenado en la atención del paciente complejo. Esto permite la continuidad de los cuidados durante períodos prolongados fuera de las salas de internación. No hallamos referencias en la bibliografía sobre la existencia de salas con estas características en el ámbito hospitalario.

Dan sustento a esta propuesta en sus primeros dos años de funcionamiento la concurrencia de los pacientes, la satisfacción que expresan y la participación interesada de las madres adolescentes en los diferentes talleres (Figuras 1, $2,3,4)$.

Un aspecto clave y el que ha sido de más difícil concreción es la selección del personal, dado que no solo revisten importancia el conocimiento y las habilidades técnicas. La capacidad de interactuar con adolescentes requiere un profundo conocimiento y aceptación

Tabla 1. Sala de Recreación para Adolescentes Internados. Hospital "Prof. Dr. J. P. Garrahan". Talleres y asistencia de pacientes. Producción 2011-2013

\begin{tabular}{lccc}
\hline & Primer año & Segundo año & Total \\
& (octubre de 2011-setiembre de 2012) & (octubre de 2012-setiembre de 2013) & n \\
\hline Asistencia de pacientes internados & 2925 & 2852 & 5777 \\
Talleres para padres adolescentes & 54 & 81 & 135 \\
Asistencia de padres adolescentes a los talleres & 602 & 782 & 1384 \\
\hline
\end{tabular}


Figura 1. Tocando piano en la Sala

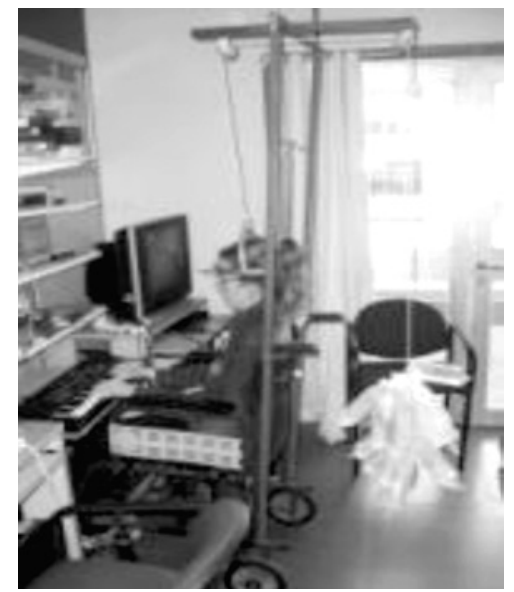

FIGURA 2. Concurrencia de padres adolescentes a los talleres

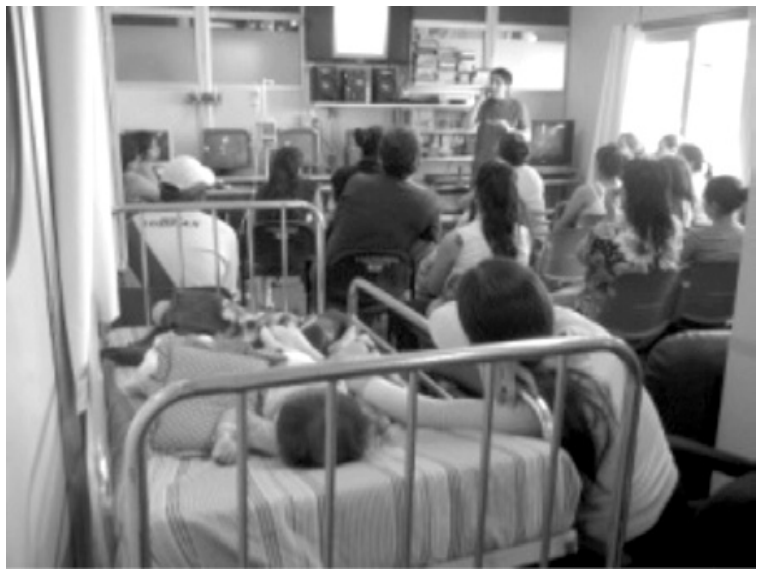

FIGURA 3. Concurrencia de una madre adolescente con su hijo enfermo a la Sala de Recreación

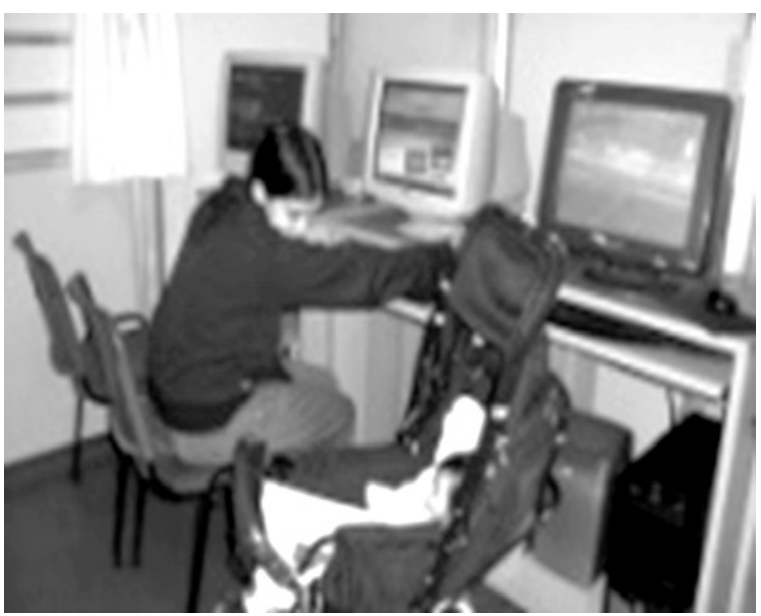

Figura 4. Jugando en la Sala

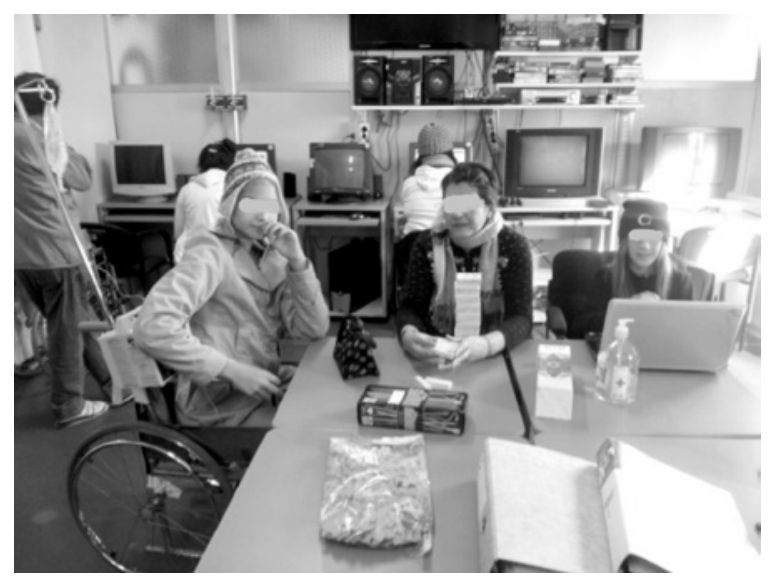

de sus características, respeto por las diferencias, capacidad de contención y una gran flexibilidad.

Por un lado, se deberán relevar indicadores y realizar encuestas que permitan evaluar en forma más precisa los resultados obtenidos. Estos estarán dirigidos a evaluar la mejora en la calidad de atención, especialmente en la reducción del estrés y el impacto negativo de la internación tanto en los pacientes con patologías agudas como en aquellos con patologías crónicas y múltiples internaciones.

Por otro lado, deberá medirse, a mediano y largo plazo, el efecto de los talleres destinados a proveer información para la prevención y definir si esta es la forma más efectiva de realizarlo.

\section{Agradecimiento}

A las enfermeras María Ester Velazquez, Mirta Durán y Norma Barraza por su capacidad puesta al servicio de los adeloscentes y su dedicación.

\section{REFERENCIAS}

1. Legislatura de la Ciudad Autónoma de Buenos Aires. Boletín Oficial de la Ciudad de Buenos Aires No 624:99599967. Ley 114: Protección Integral de los Derechos de Niños, Niñas y Adolescentes de la ciudad de Buenos Aires. Buenos Aires, 3 de febrero de 1999.

2. Dirección Nacional de Salud Materno Infantil. Ministerio de Salud de la Nación Argentina. Programa Nacional de Salud Integral en la Adolescencia. Resolución ministerial $\mathrm{N}^{\circ}$ 619/2007. 2007.

3. Programa de Salud Integral de Adolescentes y Jóvenes del Gobierno de la Ciudad de Buenos Aires. Resolución No 1751- MSGC-08. 2008.

4. Smith S. Adolescent units-an evidence-based approach to quality nursing in adolescent care. Eur J Oncol Nurs 2004;8(1):20-9.

5. Blumberg R, Devlin AS. Design issues in hospitals: the adolescent client. Environ Behav 2006;38(3):293-317.

6. Reddihough DS, Court JM. Adolescents in Hospital. J Paediatr Child Health 1979;15(3):170-2. 
7. Mato R. Encuesta para adolescentes internados. Hospital Prof. Dr. J. P. Garrahan. Septiembre-diciembre de 2008. Comunicación personal.

8. Yang NH, Dharmar M, Hojman NM, Sadorra CK, et al. Videoconferencing to reduce stress among hospitalized children. Pediatrics 2014;134(1):e169-75.

9. Liu CY, Xu L, Zang YL. Effectiveness of audiovisual interventions on stress responses in adolescents with ENT surgery in hospital: randomized controlled trial protocol. J Adv Nurs 2014;70(6):1414-24.

10. Canadian PaediatricSociety, Adolescent Health Committee. Issues of care for hospitalized youth. Paediatr Child Health 2008;13(1):61-64.

11. The Society for Adolescent Health and Medicine. Sexual and reproductive health care: a position paper of the Society for Adolescent Health and Medicine. J Adolesc Health 2014;54(4):491-6. 